\title{
Erratum to: A guideline for designing experimental studies in marketing research and a critical discussion of selected problem areas
}

\author{
Nicole Koschate-Fischer $\cdot$ Stephen Schandelmeier
}

Published online: 18 July 2014

(C) Springer-Verlag Berlin Heidelberg 2014

\section{Erratum to: J Bus Econ \\ DOI 10.1007/s11573-014-0708-6}

Figure 3 of the original article was incorrectly translated. It was supposed to be "one-factor analysis of (co-)variance" (instead of "univariate analysis of (co)variance") and "multi-factor analysis of (co-)variance" (instead of "multivariate analysis of (co-)variance").

Corrected version of Fig. 3 from Koschate-Fischer/Schandelmeier 2014:

\begin{tabular}{|c|c|c|c|}
\hline & & Design Type & \\
\hline Independent Variable & & $\begin{array}{l}\text { Between-Subjects } \\
\text { Design }\end{array}$ & $\begin{array}{l}\text { Within-Subjects } \\
\text { Design/ Mixed Design }\end{array}$ \\
\hline \multirow{2}{*}{$\begin{array}{l}\text { One Independent } \\
\text { Variable }\end{array}$} & $\begin{array}{l}\text { Two Experimental } \\
\text { Conditions }\end{array}$ & $\begin{array}{l}t \text {-Test } \\
\text { (One-Factor Analysis of } \\
(\text { Co-)Variance) }\end{array}$ & $\begin{array}{l}\text { Correlated } t \text {-Test } \\
\text { (One-Factor Analysis of } \\
\text { (Co-)Variance with } \\
\text { Repeated Measurements) }\end{array}$ \\
\hline & $\begin{array}{l}\text { Two or More } \\
\text { Experimental Conditions }\end{array}$ & $\begin{array}{l}\text { One-Factor Analysis of } \\
\text { (Co-)Variance }\end{array}$ & $\begin{array}{l}\text { One-Factor Analysis of } \\
\text { (Co-)Variance with } \\
\text { Repeated Measurements }\end{array}$ \\
\hline $\begin{array}{l}\text { More than One } \\
\text { Independent Variable }\end{array}$ & & $\begin{array}{l}\text { Multi-Factor Analysis of } \\
\text { (Co-)Variance }\end{array}$ & $\begin{array}{l}\text { Multi-Factor Analysis of } \\
\text { (Co-)Variance with } \\
\text { Repeated Measurements }\end{array}$ \\
\hline
\end{tabular}

Fig. 3 Specifying the type of ANOVA (based on Hair et al. 2010)

The online version of the original article can be found under doi:10.1007/s11573-014-0708-6.

N. Koschate-Fischer $(\bowtie) \cdot$ S. Schandelmeier

Friedrich-Alexander-Universität Erlangen-Nürnberg, GfK-Lehrstuhl für Marketing Intelligence,

Lange Gasse 20, 90403 Nuremberg, Germany

e-mail: nicole.koschate-fischer@fau.de

S. Schandelmeier

e-mail: stephen.schandelmeier@gmail.com 